\section{Visual acuity testability and comparability in Australian preschool children: The Sydney Paediatric Eye Disease Study}

JF Leone1, GA Gole², P Mitchell³, A Kifley³, AS-I Pai ${ }^{3}$ and KA Rose ${ }^{1}$

\begin{abstract}
Purpose To establish standardised protocols for vision screening, testability and comparability of three different vision tests were examined in a population-based, cross-sectional sample of preschool children (Sydney Paediatric Eye Disease Study). Methods Measurement of presenting monocular distance visual acuity (VA) using the Amblyopia Treatment Study (ATS) HOTV protocol, was attempted by all (1774) children aged $\geq 24$ months. In addition, in children aged $\geq 60$ months (576), VA was also tested using the logMAR retro-illuminated HOTV or Early Treatment Diabetic Retinopathy Study (ETDRS) linear charts (CSV 1000). Children able to have both eyes tested monocularly were considered. Results Testability significantly increased with age for all VA tests. The ATS HOTV with an overall testability of $80 \%$ (females: $82 \%$, males: $78 \%$ ) was the most testable of the VA tests $(P<0.0001)$. In children aged $<3$ years testability was low $(\leq 47 \%)$ rising to $\geq 80 \%$ in children aged $\geq 3$. In children $\geq 60$ months, testability was higher for the HOTV (94\%) than the ETDRS (59\%) chart. In those that did two VA tests, mean difference of the ATS HOTV compared with the HOTV(CSV) was -0.1 , and compared with ETDRS was $-0.12(P<0.0001)$.

Conclusions Children aged $<3$ years had poor VA testability, whereas those 3 years and above were highly testable using the ATS HOTV. The HOTV (CSV) retroilluminated test was appropriate for children aged $>5$ years, and may be possible in younger children with early educational
\end{abstract}

exposure. When comparing VA measures using these tests, the higher VA attained using the ATS HOTV, needs to be taken into account.

Eye (2012) 26, 925-932; doi:10.1038/eye.2012.60; published online 13 April 2012

Keywords: visual acuity; child; preschool; vision screening; cross-sectional studies

\section{Introduction}

Vision screening is designed to detect children who have treatable ocular conditions that impair visual acuity (VA), such as refractive error, amblyopia, and strabismus. It may also detect serious but rare ocular conditions that otherwise would have gone undetected, particularly if unilateral. To prevent visual impairment, vision screening needs to be performed as early as possible before the conclusion of the period of visual plasticity. ${ }^{1}$

Preschool vision screening was found to be beneficial in lowering the prevalence of amblyopia when children were re-assessed at ages $7.5^{2}$ and 8 years. ${ }^{3}$ A randomised control trial of repetitive vision screening from 8 to 36 months compared with one screening at 36 months, also found lower prevalence of amblyopia at age 7.5 years and improved visual outcomes in amblyopic eyes in the frequently screened group. ${ }^{4}$ However, a limitation of these studies was the use of varying vision screening protocols with age, and use of vision tests that do not always conform to Snellen or logMAR principles. ${ }^{5}$ There are limited population-based studies determining the appropriateness of
'Discipline of Orthoptics, The University of Sydney, Lidcombe, NSW, Australia

${ }^{2}$ Discipline of Paediatrics and Child Health, University of Queensland, Brisbane, QLD, Australia

${ }^{3}$ Centre for Vision Research, Westmead Millennium Institute (Westmead Hospital), University of Sydney, Sydney, NSW, Australia

Correspondence: KA Rose, Discipline of Orthoptics, Faculty of Health Sciences, University of Sydney, PO Box 170, Lidcombe 1815, NSW, Australia.

Tel: + 61293519464 ; Fax: +61293519359 E-mail: kathryn.rose@ sydney.edu.au

Received: 16 December 2011

Accepted in revised form: 24 February 2012 F Published online: 13 April 2012 
standardised VA tests in the preschool age group ${ }^{6-8}$ and none have compared the VA results gained with a goldstandard linear chart.

Although testability of amblyopia treatment study (ATS) HOTV protocol has previously been reported in population based studies, $6,7,9$ this study examines the testability of three different VA tests, including the ATS HOTV, and two versions of the logMAR tests, the goldstandard Early Treatment Diabetic Retinopathy (ETDRS), or the HOTV retro-illuminated linear charts in a large population-based sample of preschool children.

\section{Materials and methods}

\section{Participants}

The Sydney Paediatric Eye Disease Study (SPEDS) is a population-based survey of vision and eye health in children aged between 6 and 78 months in Sydney, Australia, and forms part of the Sydney Childhood Eye Study that also includes school children. The Sydney metropolitan area was stratified into inner city, suburban and outer suburban geographical regions according to the Statistical Divisions determined by the Australian Bureau of Statistics. In these three regions, postcodes were stratified according to socio-economic status (SES) and randomly selected from each region to represent the range of SES and ethnicities within Sydney. The study selected a total of four post codes and enumerated 3333 age-eligible children in a door-to-door census. A total 2461 of these children (73.8\% participation rate) were examined during 2007-2009.

\section{Procedures}

Questionnaires completed by parents, provided data on the ethnicity of the child, based on the self-identified ethnic origin of both parents, using ethnic categories consistent with the Australian Standard Classification of Cultural and Ethnic Groups. ${ }^{10}$ Children with parents of differing ethnicity were placed into a mixed ethnicity category.

All children underwent a comprehensive examination performed by orthoptists and doctors trained in the study's protocol, which was similar to that developed by the Multi-Ethnic Paediatric Eye Disease Study (MEPEDS) and the Baltimore Paediatric Eye Disease Study (BPEDS). ${ }^{6,11}$ The examination included VA, ocular motility assessment, cover test, stereoacuity, colour vision, and anthropometry, followed by cycloplegic autorefraction, slit lamp anterior segment and fundus examination, including fundus photography where possible. Cycloplegic refraction was measured, using a hand-held Retinomax K-Plus 2 autorefractor (Nikon Corporation, Tokyo, Japan), and/or the Canon RK-F1 table-mounted autorefractor (RK-F1 Auto RefKeratometer; Canon, Tokyo, Japan). Streak retinoscopy was performed if Retinomax readings with confidence ratings of $\geq 8$ were not obtained in both eyes after multiple attempts.

VA testing was attempted by all children aged $\geq 24$ months using the ATS automated protocol, ${ }^{12}$ using single-surround HOTV letters presented on the electronic VA (EVA) tester ${ }^{13}$ at $3 \mathrm{~m}$ with a letter matching card (ATS HOTV, Figure 1a). The ATS HOTV protocol included a binocular pre-test at both near and at $3 \mathrm{~m}$. If the pre-tests were successful, then the ATS HOTV was commenced with one eye occluded starting with the $0.8(6 / 38)$ sized optotype. An initial screening phase determined the approximate threshold. Confirmation of this threshold was performed and determined at the level where the majority of letters were correctly identified. A brief reinforcement phase followed, and a final threshold phase was then conducted. VA was scored as the smallest optotype seen in either of the two threshold phases. VA scores were provided in $0.1 \log$ MAR increments from 1.6 $(6 / 240)$ to $-0.1(6 / 5)$.

In addition to the ATS HOTV, all children aged $\geq 60$ months were also tested with a logMAR chart (CSV-1000 vector vision, $2.44 \mathrm{~m}$ ) using either the ETDRS (ETDRS (CSV), Figure 1b), or HOTV letters using a matching letter card (HOTV (CSV), Figure 1c). The VA testing order was not randomised in order to capture testability data for the ATS HOTV comparable with the examination protocol of MEPEDS ${ }^{7}$ and BPEDS. ${ }^{6}$ After attempting vision testing using the ATS HOTV, children aged $\geq 60$ months were then asked to read all letters on the top line of the ETDRS (CSV) chart to determine knowledge of letters. If unable to identify letters, the HOTV (CSV) test plate with matching letter card was used instead. LogMAR testing protocol adopted a similar, standardised approach to the ATS HOTV of refining the threshold VA. An initial screening phase determined approximate threshold acuity, this was followed by a threshold phase, a reinforcement phase, then a final threshold phase. Testing ceased when the child incorrectly identified three or more letters on a given line. The best VA determined from each of the two threshold phases was recorded as the final VA. Threshold monocular VA was measured as the number of letters read correctly and recorded in logMAR units, with each letter worth $0.02 \log$ MAR.

Of the 1774 children aged 24 months or older, ATS HOTV testability data were available for 1746 children. Twenty-eight children had missing data owing to a brief period when the ATS HOTV EVA system was unavailable and these children completed only a $\log$ MAR test. Of the 632 children aged $\geq 60$ months, complete $\log$ MAR testability data were available for 

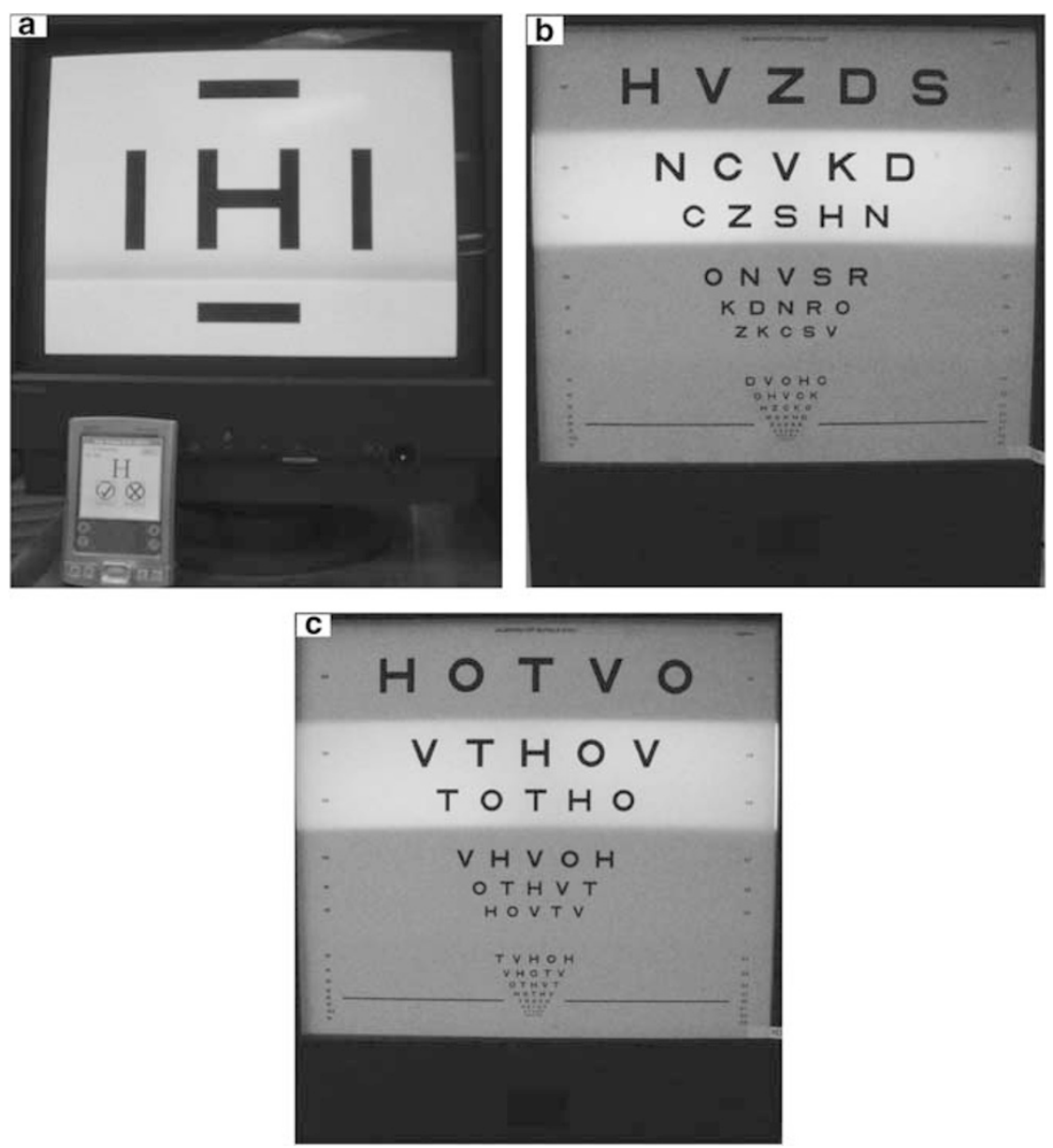

Figure 1 The visual acuity tests used in the SPEDS includes (a) the ATS HOTV visual acuity testing protocol presented on the EVA tester, (b) LogMAR chart displaying the CSV-1000 EDTRS test plate, and (c) the LogMAR chart displaying the CSV-1000 HOTV test plate.

576 children. ETDRS (CSV) VA was attempted by 548 children, of these 220 children were unable to perform test and then attempted the HOTV (CSV).

The examination procedures and informed consent forms were approved by the Human Research Ethics Committee of the University of Sydney, and adhered to the tenets of the Declaration of Helsinki. Written informed consent was obtained from the parent or guardian of each study participant before examinations. We certify that all applicable institutional and governmental regulations concerning the ethical use of human volunteers were followed during this research.

\section{Statistical analysis}

A child was defined as testable when they could complete the test monocularly in both the right and the left eye. Children who could only complete the test in one eye or unable to complete the test in both eyes were deemed non-testable. Testability was defined as the ability to complete the VA test in both eyes on the first attempt. HOTV (CSV) testability was determined by each child's ability to complete either the HOTV (CSV) or ETDRS (CSV) test, since children capable of ETDRS (CSV) testing should also be capable of HOTV (CSV) testing, but would not have been asked to complete both. Age-, gender- and ethnicity-specific testability rates were calculated. Logistic regression was used to assess associations between testability and age, using children aged 72 months or more as the reference group. Logistic regression was also used to assess associations between testability and ethnic group, using European Caucasian as the reference. Chi square tests were used to assess associations between gender and testability. Among children attempting both tests, ATS HOTV and either of the logMAR (CSV) charts, testability rates were compared using McNemar's ${ }^{14}$ test for difference in correlated proportions, and ATS HOTV as the reference test. 
Comparability between ATS HOTV and logMAR VA measurements was analysed among children who successfully completed both tests: 187 children (374 eyes) for the comparison of ATS HOTV and HOTV (CSV) and 313 children (626 eyes) for the comparison of ATS HOTV and ETDRS (CSV). Because the null expectation was zero difference between tests for any randomly chosen eye and no correlation was expected between differences in scores for eyes belonging to the same person, eye rather than person was chosen as the unit of analysis. A BlandAltman ${ }^{15}$ assessment for agreement was used to compare the two VA methods. The limits of agreement were defined as mean measurement differences \pm 2 SD and presented with $95 \%$ confidence intervals (CI). Linear regression was used to assess associations between gender, ethnic group, refractive error and the differences between VA test measurements. SAS software version 9 was used for all analyses (SAS Institute, Cary, NC, USA).

\section{Results}

Of the 2461 children who participated in SPEDS, 1774 children were aged 24 months or older, of whom $48 \%$ were female. The study examined children from a number of different ethnic groups. Children with European Caucasian ethnicity were the predominant group (46\%), 21\% were of East Asian ethnicity, and 33\% were of other and mixed ethnicities.

\section{Testability}

VA testing using the ATS HOTV protocol was attempted by 1746 of the 1774 children, with $80 \%$ able to complete the test, however, testability was strongly associated with age $(P<0.0001)$. In the youngest children, aged 24 to $<30$ months $(n=181)$, only $10 \%$ were able to be perform the test (Figure 2), although this improved markedly in the next 6 months of age $(n=230)$ to $47 \%$. The testability rate continued to increase and it was $80 \%$ by $36-<42$ months $(n=182), 93 \%$ at $42-<48$ months $(n=181)$, and reached $95 \%$ at age $48-<54$ months $(n=171)$. Testability then approached $100 \%$ in the older children; $98 \%$ at both $54-<60$ months $(n=189)$ and $60-<66$ months $(n=163)$, $99 \%$ at $66-<72$ month $(n=174)$ and was $100 \%$ at $72-<78$ months $(n=129)$. It fell slightly to $97 \%$ in the oldest group ( $\geq 78$ months, $n=146$ ) which is likely to be related to a higher percentage of children with developmental delay in this group.

There were 576 children aged $\geq 60$ months or older who attempted the HOTV (CSV) and testability also improved with increasing age $(P=0.016)$. In the age group $\geq 60$ months, testability measures for both the ATS HOTV and HOTV (CSV) were above 90\%. Further, 548 children aged $\geq 60$ months attempted the ETDRS (CSV),

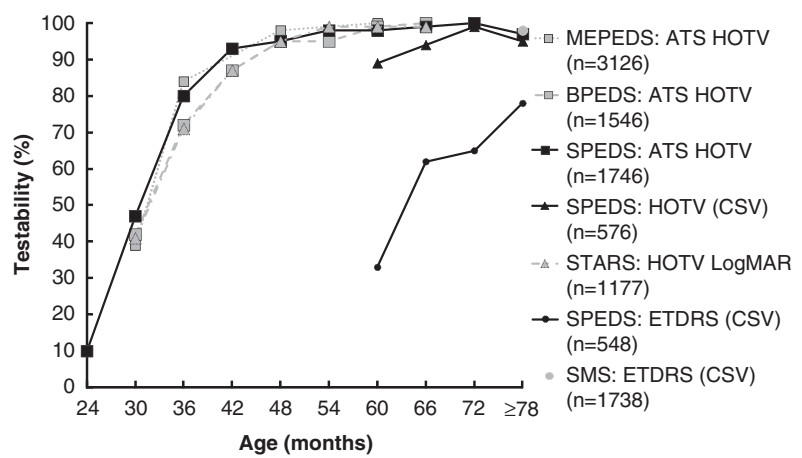

Figure 2 Testability of VA (ATS HOTV, HOTV (CSV), HOTV (logMAR), EDTRS (CSV)) in children from five studies (MEPEDS, BPEDS, SPEDS, STARS, SMS). Note: Testability was presented in 6-month age groups for all studies 6,8,17 except for MEPEDS, ${ }^{7}$ where testability was presented in 1 year increments above 36 months of age. SMS mean age was 80.4 months (range 66 to 100.8 months), testability rate for the entire age range is presented only. Presenting testability rates were calculated for all studies, note BPEDS presenting testability was only reported for African Americans and European Caucasian populations and not the total population tested.

of whom $328(60 \%)$ were able to complete testing. Testability for the ETDRS (CSV) also improved with increasing age $(P<0.0001)$, but did not reach $100 \%$ in any of the age groups, and was $79 \%$ in the oldest group (age $\geq 78$ months). There were six children older than 60 months who were unable to complete any VA test. Of these, two had VA $<6 / 120$, and the other children did not have the capacity to complete the test because of developmental delay and/or autism spectrum disorders.

The proportion of children aged $\geq 60$ months who completed the ATS HOTV protocol (reference test) was compared with the proportion who completed either the HOTV or ETDRS (CSV). The testability of the ATS HOTV was not significantly different to the HOTV (CSV) in children aged $\geq 72$ months (Table 1 ), but was significantly higher than the ETDRS (CSV) for all age groups.

There was a general trend for girls to achieve higher testability outcomes in those VA tests using HOTV letters with matching cards than for boys (Table 2). However, this was statistically significant only for the ATS HOTV between the ages of 42 to $<60$ months $(P=0.0002)$, and for the HOTV (CSV) aged $\geq 60$ months $(P=0.017)$.

Children of 'other' ethnic groups generally had lower testability than children of European Caucasian ethnicity for the tests using matching cards, but this was statistically significant for only the ATS HOTV test in the youngest age group ( $24-<42$ months) $(P=0.005)$. Across all of the vision tests, there was a general trend for 
Table 1 Comparison of the Testability across ATS HOTV, EDTRS (CSV), and HOTV (CSV) Vision Tests by Age in children $\geq 60$ months

\begin{tabular}{|c|c|c|c|c|c|c|c|c|}
\hline $\begin{array}{l}\text { Age group } \\
\text { (months) }\end{array}$ & $\begin{array}{l}\text { Total attempted } \\
\qquad \mathrm{n}^{\mathrm{a}}\end{array}$ & $\begin{array}{c}\text { ATS HOTV } \\
\quad \% \text { able })\end{array}$ & $\begin{array}{c}\text { ETDRS (CSV) } \\
(\% \text { able })\end{array}$ & $\mathrm{P}$ value & $\begin{array}{c}\text { Total Attempted } \\
\mathrm{n}^{\mathrm{a}}\end{array}$ & $\begin{array}{c}\text { ATS HOTV } \\
\quad(\% \text { able })\end{array}$ & $\begin{array}{l}\text { HOTV (CSV) } \\
\quad(\% \text { able })\end{array}$ & P value \\
\hline 60 to $<66$ & 129 & $97 \%$ & $33 \%$ & $<0.0001$ & 134 & $97 \%$ & $90 \%$ & 0.002 \\
\hline 66 to $<72$ & 155 & $99 \%$ & $62 \%$ & $<0.0001$ & 163 & $99 \%$ & $94 \%$ & 0.003 \\
\hline 72 to $<78$ & 119 & $100 \%$ & $65 \%$ & $<0.0001$ & 124 & $100 \%$ & $99 \%$ & $\sim 1$ \\
\hline$\geq 78$ & 131 & $98 \%$ & $78 \%$ & $<0.0001$ & 135 & $98 \%$ & $96 \%$ & 0.3 \\
\hline$\geq 60$ (Total) & 534 & $99 \%$ & $59 \%$ & $<0.0001$ & 556 & $99 \%$ & $94 \%$ & $<0.0001$ \\
\hline
\end{tabular}

${ }^{\mathrm{a}} n=$ total who attempted two vision test either ATS HOTV and ETDRS (CSV), or ATS HOTV and HOTV (CSV). ${ }^{\mathrm{b}}$ Reference test. Bold $P$ values indicate statistical significance.

Table 2 Testability of the ATS HOTV, HOTV (CSV) a , and EDTRS (CSV) vision tests by age, gender and ethnicity

\begin{tabular}{|c|c|c|c|c|c|c|c|c|}
\hline \multirow[b]{3}{*}{ Months } & \multicolumn{3}{|c|}{ Gender } & \multicolumn{5}{|c|}{ Ethnicity } \\
\hline & \multirow{2}{*}{$\frac{\text { Male }}{\mathrm{n}^{\mathrm{b}}(\% \text { able })}$} & \multicolumn{2}{|c|}{ Female } & \multirow{2}{*}{$\frac{\text { European Caucasian (ref) }}{\mathrm{n}^{\mathrm{b}}(\% \text { able })}$} & \multicolumn{2}{|c|}{ East Asian } & \multicolumn{2}{|c|}{ Other } \\
\hline & & $\mathrm{n}^{\mathrm{b}}(\%$ able $)$ & P value & & $\mathrm{n}^{\mathrm{b}}(\%$ able $)$ & $\mathrm{P}$ value & $\mathrm{n}^{\mathrm{b}}(\%$ able $)$ & $\mathrm{P}$ value \\
\hline \multicolumn{9}{|l|}{ ATS HOTV } \\
\hline 24 to $<42$ & $316(44 \%)$ & $277(47 \%)$ & 0.5 & $278(49 \%)$ & $123(53 \%)$ & 0.5 & $192(36 \%)$ & 0.005 \\
\hline 42 to $<60$ & $274(92 \%)$ & $267(99 \%)$ & 0.0002 & $246(96 \%)$ & $111(98 \%)$ & 0.3 & $184(93 \%)$ & 0.3 \\
\hline$\geq 60$ & $319(98 \%)$ & $293(99 \%)$ & 0.4 & $275(99 \%)$ & $138(100 \%)$ & NS & $199(97 \%)$ & 0.1 \\
\hline \multicolumn{9}{|c|}{ ETDRS (CSV) } \\
\hline$\geq 60$ Total & $291(57 \%)$ & $257(63 \%)$ & 0.2 & $238(56 \%)$ & $126(69 \%)$ & 0.015 & $184(59 \%)$ & 0.6 \\
\hline \multicolumn{9}{|c|}{ HOTV $(C S V)^{\mathbf{b}}$} \\
\hline$\geq 60$ Total & $308(93 \%)$ & $268(97 \%)$ & 0.017 & $255(95 \%)$ & $133(99 \%)$ & 0.050 & $188(91 \%)$ & 0.2 \\
\hline
\end{tabular}

a Based on the assumption that those who performed the EDTRS (CSV) are also able to perform the HOTV (CSV), and therefore are included in this testable group. ${ }^{\mathrm{b}} n=$ total who attempted vision test within the specific age and ethnicity or gender group. Bold $P$ values indicate statistical significance.

children of East Asian ethnicity to have higher testability rates (Table 2). This was marginally significant for HOTV (CSV) $(P=0.05)$ and was statistically significant for the ETDRS (CVS) test $(P=0.015,1.7$ OR $(95 \% \mathrm{CI})$ : 1.1-2.7).

\section{VA Comparisons $(\geq 60$ months)}

In right eyes of children $\geq 60$ months, the mean uncorrected VA for the ATS HOTV was $0.02 \operatorname{logMAR}$ (6/6-1) (95\% CI: 0.01-0.03), for the HOTV (CSV) was 0.13 (6/7.5-2) (0.11-0.14), and for the ETDRS (CSV) was 0.13 (6/7.5-2) (0.12-0.14). Corresponding figures for the left eyes of children $\geq 60$ months were 0.01 (6/6-1) (0.01-0.02), 0.13 (6/7.5-2) (0.12-0.15), and 0.13 (6/7.5-2) (0.12-0.14) letters, respectively. The difference between eyes for each test was not statistically significant $(P=0.5$, $0.7,0.6$ respectively). The VA between the two eyes for each test was well correlated (Spearman rank correlation: $0.8,0.8,0.7$ respectively). Data for both eyes of children $\geq 60$ months were therefore pooled, for a total of 374 observations for the HOTV (CSV), and 626 observations for the ETDRS (CSV).
When VA measured using the ATS HOTV was compared with the VA attained using the HOTV (CSV), there was a significant difference in VA $(P<0.0001)$. On average this difference was $-0.1 \log$ MAR $(95 \%$ CI: -0.09 to -0.11 ), that is, one line higher using the ATS HOTV. The $95 \%$ limits of agreement between the two tests ranged from -0.22 (95\% CI: -0.21 to -0.24$)$ to 0.04 (95\% CI: 0.03 to 0.05 ) (Figure 3a). This difference was not associated with age, gender, refractive error, or ethnicity.

Similarly, when comparing VA performed using the ETDRS (CSV) with the ATS HOTV $(n=626)$, there was a mean -0.12 logMAR ( 1 line +1 letter) difference in VA $(P<0.0001,95 \%$ CI: -0.12 to -0.13$)$ and the $95 \%$ limits of agreement ranged from -0.27 (95\% CI: -0.26 to -0.28 ) to 0.019 (95\% CI: 0.01 to 0.03 ) (Figure 3b). This difference was associated with age $(P=0.04)$, but was not associated with gender, refractive error, or ethnicity.

In children aged $\geq 60$ months, the ATS HOTV compared with either of the logMAR charts did not consistently provide similar measurements for VA and included clinically important discrepancies of $>-0.1$ $\log$ MAR. This discrepancy was present in $30 \%$ of the 
a
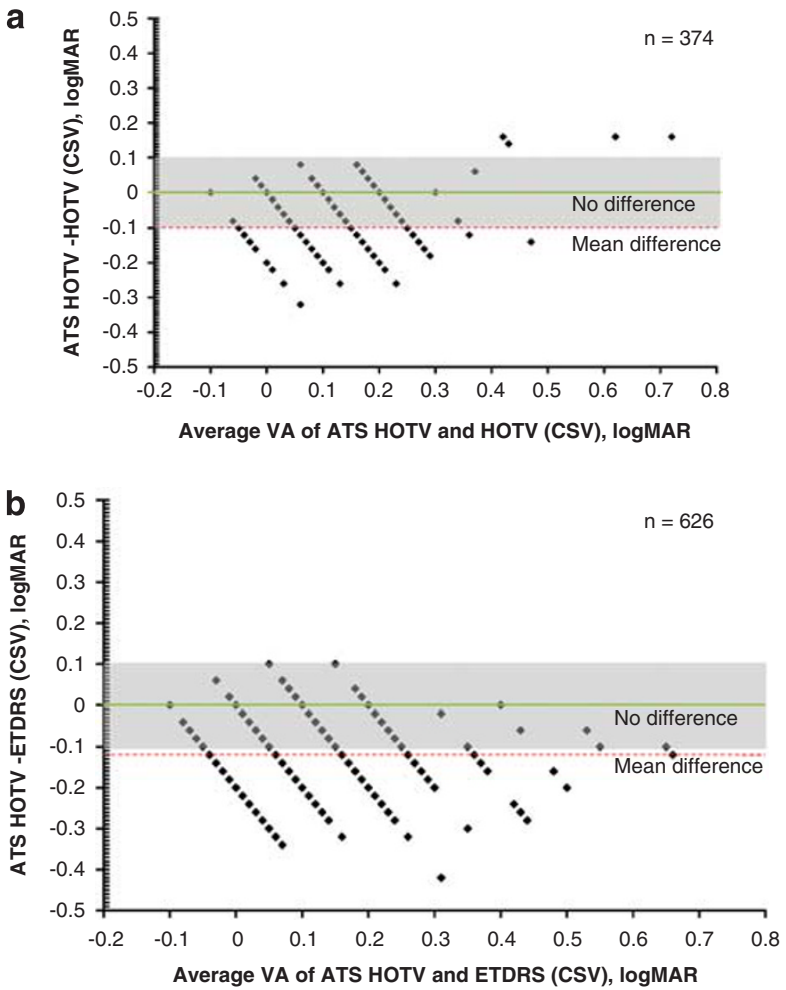

Figure 3 Bland-Altman plots of VA obtained using: (a) ATS HOTV compared with HOTV (CSV); (b) ATS HOTV compared with ETDRS (CSV). Grey shaded area indicates an area \pm 0.1 $\log$ MAR, of clinically non-significant difference between the two tests. Note: Negative score on the y axis indicates child recorded a better VA using the ATS HOTV.

cases for HOTV (CSV), and 56\% of the cases for ETDRS (CSV).

\section{Discussion}

The testability of VA in young children is important when developing recommendations for preschool vision screening protocols, as well as determining ageappropriate clinical tests. We evaluated the testability of the single surround ATS HOTV protocol in a large population-based sample of Australian preschool children. In a subset of children aged $\geq 60$ months, we also established the testability of linear HOTV (CSV) or ETDRS (CSV) logMAR charts and compared these VA measures with those obtained using the ATS HOTV. We have identified two age-based protocols that may be suitable for vision screening of preschool children. The first is the ATS HOTV protocol with high testability $(\geq 80 \%)$ in children aged $3-5$ years. The second is the linear HOTV (CSV) with high testability ( $\geq 90 \%)$ in children aged $>5$ years. This may also be more appropriate as it is more aligned to the gold standard ETDRS. It is to be noted that girls were more testable than boys overall and this reached significance in some agegroups. However, this occurred when testability was already in excess of $90 \%$ and therefore would have little impact when choosing tests. Our findings suggest that the ATS HOTV could not be recommended as a suitable vision test for screening children aged 2-3 years, due to low rates of testability. However, this age group is not a target age for vision screening protocols. ${ }^{16}$

We did not perform HOTV (CSV) testing in children under the age of 5 years. The STARS study ${ }^{8}$ that exclusively used HOTV (CSV) in children as young as 30 months, found that testability reached $\geq 80 \%$ in children as young as 3-3.5 years. However, this result may be population-specific. An age-matched comparison of testability for children aged $>5$ years in our study $(92 \%)$ with the same age group in STARS (99\%), shows greater testability in the STARS sample (Figure 2). However, for East Asian children in our sample, the difference in testability was reduced. The higher testability of East Asian children at this age may be due to early learning strategies used by parents, rather than East Asian ethnicity per se.

VA testability using the ETDRS (CSV) vision chart was low and did not achieve a rate of $80 \%$ even in the oldest children. When this is compared with the Sydney Myopia Study ${ }^{17}$ (SMS), children of a similar age performing the same test, achieved a testability of $98 \%$. Although SMS children attempted only one VA test (usually ETDRS), they had also 2 years of schooling and the SPEDS children were not yet at this level of schooling. Overlap of ages with different levels of schooling occur when parents vary the age of school enrolment by up to 1 year in formal education, this would appear to have a major influence on VA testability in children in this age group.

In determining suitable protocols for vision screening, it is also important to establish if there are any differences in the VA outcomes for different VA tests. Comparison of the VA measures using these three tests suggests that caution is required when comparing the results from a single surround letter optotype vision test such as the ATS HOTV to the outcomes using linear charts. There was at least a mean full line increase in VA measured using the ATS HOTV, when compared with VA measured with the HOTV or ETDRS (CSV), in the same children. It is to be noted that the ATS HOTV conforms to the correct principles of optotype design, having crowding bars optimally placed at half the optotype height from the letter optotype. ${ }^{12,18}$ Although detecting intra-ocular difference in VA screening for amblyopia will not be affected by the difference in VA outcomes using the different tests, it will have some impact on referral cut-offs for binocular reduced VA such as what occurs with bilateral disorders such as refractive errors. 
Other studies, analysing test-retest scores using the ATS HOTV, have observed difference in scores between the two presentations of the same test. These studies have identified a difference of $0.2 \log$ MAR levels or more in VA measures to be clinically significant. ${ }^{12,13}$ The mean difference between the ATS HOTV and the HOTV or ETDRS (CSV) observed in our study was less than this, which implies that this may not be significant. However, there was large variability in the difference in VA observed in some individuals, ranging up to 3 to 4 lines difference between the two types of test, which may be due to the high variability that has been found in testretest of even the same VA test in some children. ${ }^{12,13}$ The referral criteria from vision screening tests may still need to be set at different levels according to whether using single surround or linear optotypes. It has been shown that single optotypes (without crowding) can underestimate the degree of amblyopia compared with linear charts. ${ }^{19}$ However, optimally designed single surround optotypes such the ATS HOTV have been shown not to underestimate amblyopia in school aged children, ${ }^{18}$ although this may need to be systematically investigated in children of preschool age.

It could be argued that a limitation of the comparability results of our study is the test order, with the ATS HOTV routinely tested first. This may have provided an opportunity to improve VA scores on the second test, either the HOTV or ETDRS (CSV), by learning familiarisation and through repetition. If this did occur, then randomisation of test order may have produced an even greater difference between the ATS and linear-based tests. This needs to be systematically examined. Conversely, it could be argued that the reduction in the second VA measure was due to fatigue and here randomisation would have reduced the difference. It is, however, to be noted that from the 6-year-old sample of the SMS, in which the ETDRS (CSV) was done alone, the mean VA (49.6 letters) ${ }^{17}$ was not statistically significantly different from the VA result in our sample in children from the same age group ( $\geq 66$ months, mean: 48.9 letters, $P=0.84$ ) using the linear-based chart. This suggests that test order in SPEDS may not have negatively impacted the VA outcome using the ETDRS or HOTV (CSV). This may imply that the higher level of VA obtained with the ATS HOTV test could be related to children finding it a cognitively easier test to perform and/or owing to the inherent differences in the presentation of the letters, with more complex contour interactions inherent in linear charts.

The VA tests in our study were conducted by highly motivated and trained eye professionals with paediatric specialisation. Although we did not compare testability using trained and untrained personnel, our results may indicate the upper limit of testability of VA for children of this age. This, however, needs to be systematically tested.

Our study has shown that ATS HOTV is the most testable of the three VA charts for the target preschool vision screening age-group of 3 to 5 years. This result is consistent with findings from the MEPEDS ${ }^{7}$ and the BPEDS. ${ }^{6}$ The use of charts with optotypes arranged linearly as opposed to single surround optotypes may be optimal for children aged $>5$ years, or earlier in specific populations of children who have had educational exposure at an earlier age. The role of education and the potential role of pre-training and their effects on levels of testability in preschool children needs further systematic examination in a preschool vision screening setting.

\section{Summary}

\section{What was known before}

- High testability rates for the use of the Amblyopia Treatment Study (ATS) HOTV protocol in testing vision of preschool children ( $\geq 3$ years) has been previously reported in African American, Hispanic, and White populations in the US (MEPEDS and BPEDS), and we have found similar levels of testability in an ethnically diverse population in Australia.

What this study adds

- In this study we compare the testability of adult-standard ETDRS and HOTV vision tests in children $\geq 60$ months with that of the ATS HOTV protocol.

- We found that, although the ATS HOTV has the highest testability age-for-age, the testability of the linear HOTV optotypes is $90 \%$ and above in these older children, but testability using the EDTRS is lower overall.

- The children of East Asian ethnicity, however, had higher testability using the adult ETDRS than children of other ethnicities, which may be related to early education practices in this ethnic group.

- This study uniquely compares visual acuity in preschool children measured using both the ATS HOTV protocol and the ETDRS or HOTV charts, with a mean one line difference in visual acuity measured by the two methods, single surround optoypes versus linear presentation.

\section{Conflict of interest}

The authors declare no conflict of interest.

\section{Acknowledgements}

The Sydney Paediatric Eye Disease Study was supported by the National Health \& Medical Research Council (NHMRC), Australia, grant no. 402425, 2007-9. The Australian Research Council (CEO561903). J Leone holds 
a NHMRC Postgraduate Research Scholarship (Scholarship ID 457173).

\section{References}

1 Jastrzebski GB, Hoyt CS, Marg E. Stimulus deprivation amblyopia in children. Sensitivity, plasticity, and elasticity (SPE). Arch Ophthalmol 1984; 102(7): 1030-1034.

2 Williams C, Northstone K, Harrad RA, Sparrow JM, Harvey I. Amblyopia treatment outcomes after preschool screening v school entry screening: observational data from a prospective cohort study. Br J Ophthalmol 2003; 87(8): 988-993.

3 Eibschitz-Tsimhoni M, Friedman T, Naor J, Eibschitz N, Friedman Z. Early screening for amblyogenic risk factors lowers the prevalence and severity of amblyopia. J AAPOS 2000; 4(4): 194-199.

4 Williams C, Northstone K, Harrad RA, Sparrow JM, Harvey I. Amblyopia treatment outcomes after screening before or at age 3 years: follow up from randomised trial. BMJ 2002; 324(7353): 1549.

5 Fern KD, Manny RE. Visual acuity of the preschool child: a review. Am J Optom Physiol Opt 1986; 63(5): 319-345.

6 Friedman DS, Repka MX, Katz J, Giordano L, Ibironke J, Hawes $\mathrm{P}$ et al. Prevalence of decreased visual acuity among preschool-aged children in an American urban population: the Baltimore Pediatric Eye Disease Study, methods, and results. Ophthalmology 2008; 115(10): 1786-1795.

7 Cotter SA, Tarczy-Hornoch K, Wang Y, Azen SP, Dilauro A, Borchert $\mathrm{M}$ et al. Visual acuity testability in AfricanAmerican and Hispanic children: the multi-ethnic pediatric eye disease study. Am J Ophthalmol 2007; 144(5): 663-667.

8 Trager MJ, Dirani M, Fan Q, Gazzard G, Selvaraj P, Chia A et al. Testability of vision and refraction in preschoolers: the strabismus, amblyopia, and refractive error study in singaporean children. Am J Ophthalmol 2009; 148(2): 235-241; e236.

9 Friedman DS, Repka MX, Katz J, Giordano L, Ibironke J, Hawse $\mathrm{P}$ et al. Prevalence of amblyopia and strabismus in
White and African American children aged 6 through 71 Months The Baltimore Pediatric Eye Disease Study. Ophthalmology 2009; 116(11): 2128-2134; e1-2.

10 Australian Bureau of Statistics. Australian Standard Classification of Cultural and Ethnic Groups (ASCCEG). Available at: http://www.ausstats.abs.gov.au/Ausstats / subscriber.nsf/0/00A57A2C8FE19CACCA2570360074713B / $\$$ File/12490_2005.pdf (accessed 29 March 2012).

11 Varma R, Deneen J, Cotter S, Paz SH, Azen SP, Tarczy-Hornoch $\mathrm{K}$ et al. The multi-ethnic pediatric eye disease study: design and methods. Ophthalmic Epidemiol 2006; 13(4): 253-262.

12 Holmes JM, Beck RW, Repka MX, Leske DA, Kraker RT, Blair RC et al. The amblyopia treatment study visual acuity testing protocol. Arch Ophthalmol 2001; 119(9): 1345-1353.

13 Moke PS, Turpin AH, Beck RW, Holmes JM, Repka MX, Birch EE et al. Computerized method of visual acuity testing: adaptation of the amblyopia treatment study visual acuity testing protocol. Am J Ophthalmol 2001; 132(6): 903-909.

14 McNemar Q. Note on the sampling error of the difference between correlated proportions or percentages. Psychometrika 1947; 12(2): 153-157.

15 Bland MJ, Altman DG. Statistical methods for assessing agreement between two methods of clinical measurement. Lancet 1986; 327(8476): 307-310.

16 Donahue SP. The relationship between anisometropia, patient age, and the development of amblyopia. Trans Am Ophthalmol Soc 2005; 103: 313-336.

17 Robaei D, Rose K, Ojaimi E, Kifley A, Huynh S, Mitchell P. Visual acuity and the causes of visual loss in a populationbased sample of 6-year-old Australian children. Ophthalmology 2005; 112(7): 1275-1282.

18 Stager DR, Everett ME, Birch EE. Comparison of crowding bar and linear optotype acuity in amblyopia. Am Orthoptic J 1990; 40: 51-56.

19 Simmers AJ, Gray LS, Spowart K. Screening for amblyopia: a comparison of paediatric letter tests. Br J Ophthalmol 1997; 81(6): 465-469. 